\title{
Motion-induced blindness measured objectively
}

\author{
Peter Kramer • Stefano Massaccesi - Luca Semenzato • \\ Stefano Cecchetto $\cdot$ Paola Bressan
}

Published online: 31 August 2012

(C) Psychonomic Society, Inc. 2012

\begin{abstract}
During central fixation, a moving pattern of nontargets induces repeated temporary blindness to even salient peripheral targets: motion-induced blindness (MIB). Hitherto, behavioral measures of MIB have relied on subjective judgments. Here, we offer an objective alternative that builds on earlier findings regarding the effects of MIB on the detectability of physical target offsets. We propose a small modification of regular MIB displays: Following a variable duration (lead time), one of the targets is physically removed. Subjects are to respond immediately afterward. We hypothesize that illusory target offsets, caused by MIB, are mistaken for physical target offsets and that errors should thus increase with lead time. Indeed, for both nonsalient and salient targets, we found that detection accuracy for physical target offsets dramatically decreased with lead time. We conclude that target offset detection accuracy is a valid objective measure of MIB. With our method, effects of guessing are minimal, and the fitting of psychometric functions is straightforward. In principle, a staircase extensionfor more efficient data collection-is also possible.
\end{abstract}

Keywords Motion-induced blindness - Objective measure Perceptual filling-in $\cdot$ Awareness

Electronic supplementary material The online version of this article (doi:10.3758/s13428-012-0246-5) contains supplementary material, which is available to authorized users.

P. Kramer $(\bowtie) \cdot$ S. Massaccesi $\cdot$ L. Semenzato $\cdot$ S. Cecchetto

P. Bressan

Dipartimento di Psicologia Generale, Università di Padova,

Via Venezia 8,

35131 Padova, Italy

e-mail: peter.kramer@unipd.it

\section{Introduction}

If central fixation is maintained, a moving pattern of nontarget shapes (henceforth, inducers) provokes repeated temporary blindness to even salient peripheral target shapes - that is, motion-induced blindness (MIB; Bonneh, Cooperman, \& Sagi, 2001). The effect is most likely due to a failure of awareness (Donner, Sagi, Bonneh, \& Heeger, 2008; Klotz \& Ansorge, 2007; Mitroff \& Scholl, 2004, 2005; MontaserKouhsari, Moradi, Zandvakili, \& Esteky, 2004) involving the thalamus (Wilke, Mueller, \& Leopold, 2009) and various areas of the visual cortex (Donner et al., 2008; Libedinsky, Savage, \& Livingston, 2009; Schölvinck \& Rees, 2009).

So far, the behavioral measurement of MIB has relied on subjective judgments that are usually provided with buttonpresses. Two measures can be found in the literature (and in the related literature on perceptual filling-in): (1) the time until the target is judged to disappear for the first time and (2) the proportion of the trial during which the target is judged invisible. The two measures tend to lead to similar results (Hsu, Yeh, \& Kramer, 2004). In the present study, we seek an objective version of the first measure. In this version, unlike in subjective ones, responses are verifiably correct or incorrect, and subjects are given the opportunity to improve with feedback.

Our method builds on previous investigations of the effects of MIB on the detectability of physical target offsets. Whereas physical onsets during MIB tend to disrupt the phenomenon (Kawabe, Yamada, \& Miura, 2007), physical offsets do not and tend to go unnoticed. In general, physical offsets produce a different physiological response than do illusory offsets (Donner et al., 2008). If, however, under suitable conditions, they occur during illusory offsets (caused by MIB), then physical offsets do not trigger the N1 event-related potential that is normally observed with physical offsets and produce only a weak $\mathrm{P} 3$ potential that is 
attributed to a type of aftereffect (Klotz \& Ansorge, 2007). The suitable conditions are those in which the contrast between the targets and the background is not too high and the targets are sufficiently far away from fixation (and, we add, not too large). Moreover, under these conditions, physical offsets produce not only a minimal physiological response, but also a minimal behavioral one. That is, they do not reach awareness (Klotz \& Ansorge, 2007; MontaserKouhsari et al., 2004; cf. Mitroff \& Scholl, 2004).

Hitherto, as far as we know, MIB has only been measured subjectively, never objectively. Research on the effects that MIB has on the detectability of physical target offsets is no exception; subjects indicate the onset of MIB with a buttonpress on the basis of their personal impression, which is unverifiable and neither correct nor incorrect. Unlike MIB, the detectability of physical offsets is, instead, measured objectively; if subjects indicate that an offset has occurred and it did occur, they are correct; otherwise, they are incorrect. If one's goal does not concern the detectability of physical target offsets during MIB, but the characteristics of MIB itself (how it varies with such factors as stimulus duration, target contrast, perceptual grouping, or even some particular mental disorder; Tschacher, Schuler, \& Junghan, 2006), then physical target offsets could be used to objectively measure MIB.

More specifically, we argue that only a small modification of regular MIB displays is needed to allow a very simple, straightforward, and objective measurement of MIB. Unlike the usual methods, ours involves the physical removal of one of the targets (or the only target, if there is only one) after a variable duration (henceforth, lead time; see Supplementary Materials). The subjects' task is to respond immediately after this removal. In this case, the response is correct. In the case of an anticipation or missed response deadline, the response is incorrect. The task involves an extremely simple go/no-go decision: When the target disappears, respond; otherwise, wait. We hypothesize that the probability of an illusory target offset, due to MIB, increases with lead time. This, in turn, should reduce the probability of detecting the target's physical offset. That is, we argue that the accuracy in detecting the physical target offset can be taken as an objective measure of MIB, and we predict that this accuracy will decrease with lead time.

Intuitively, targets with a low contrast with respect to the background disappear from view earlier, and for a longer duration, than targets with a moderately high contrast (Hsu et al., 2004). Counterintuitively, however, so do targets with a high contrast (Bonneh et al., 2001; Hsu et al., 2004). That is, MIB is a U-shaped function of target contrast. The illusory disappearance of salient, high-contrast targets surprises naïve observers, who often find it hard to believe that, physically, these targets are still present. The saliency effect may be due not to contrast, but to a concurrent effect of perceptual grouping between target and inducers (Hsu et al.,
2004). Nonetheless, it is a striking feature of MIB. If our objective method of measuring MIB works as predicted, we should be able to replicate MIB with salient targets and, ideally, find a larger MIB effect with these salient targets than with nonsalient, but still well-visible, ones.

Here, more specifically, we investigated whether on a gray background, containing dark-gray moving inducers, more MIB (lower accuracy) would be observed with a white target disk (salient) than with a black one (well visible, but less salient; Fig. 1; see Supplementary Materials). The white target disk has not only a higher contrast with the background as whole (including the inducers) than does the black disk, but also an opposite luminance polarity with respect to the inducers. That is, whereas the luminance of the white disk is higher than that of the background, the luminance of the inducers is lower. This circumstance should produce poorer grouping between the white target and the inducers than between the black target and the inducers (Masin, 2003; see also Bressan \& Kramer, 2008). The differential grouping strengths, in turn, should result in more MIB for the white than for the black target (Hsu et al., 2004).

A final step to ensure success involves enforcing proper fixation. Monitoring eye movements during MIB is uncommon. Asking experienced subjects not to move their eyes is usually enough to prevent them from fixating a target. Because targets are typically presented peripherally, far away from fixation, subjects tend to notice when they have (involuntarily) moved their eyes toward a target and can

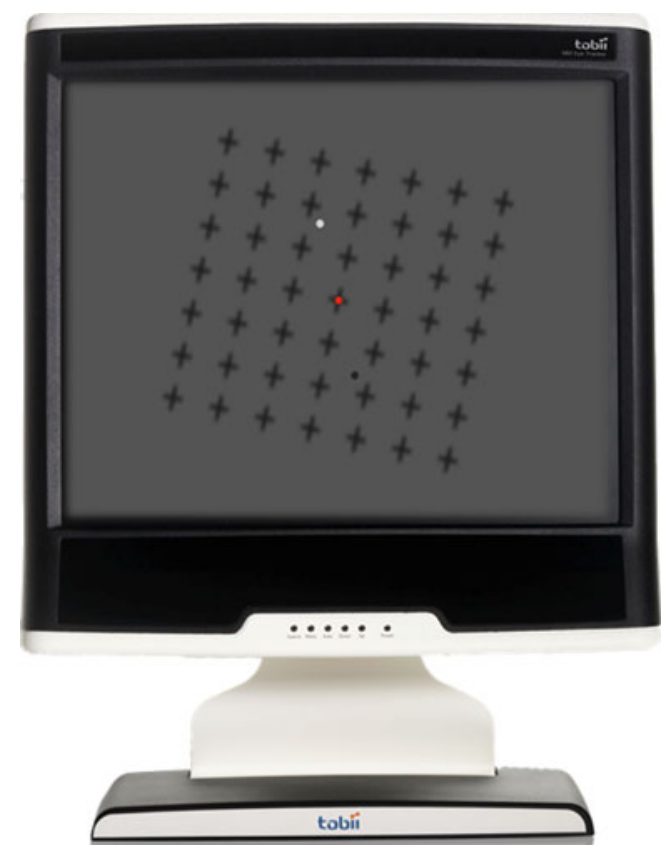

Fig. 1 The stimulus on a monitor with a built-in Tobii eyetracker in the black rectangular area immediately below the screen. The red disk is the fixation spot. The pattern of crosses (MIB inducers) rotates at 3.48 rotations $/ \mathrm{s}$. The black and white disks are the targets. After a variable lead time, one of them is removed at random 
then warn the experimenter about it. Although on average it is weakened by microsaccades, MIB can occur in their presence (Bonneh et al., 2010). However, we informally noticed that MIB is completely eliminated by continuously moving one's eyes back and forth throughout the trial, even if these eye movements are not directed toward a target. Whereas low-level retinotopically specific adaptation is not critical to MIB (Mitroff \& Scholl, 2004; Montaser-Kouhsari et al., 2004), this finding suggests that adaptation or inhibition (Gorea \& Caetta, 2009; Hsu, Yeh, \& Kramer, 2006) at a higher, non-retinotopically-specific level does play a crucial role. This is also suggested by the fact that MIB is never instantaneous. Therefore, to avoid any confounding effects of eye movements, we monitored them and excluded trials that contained them.

\section{Method}

\section{Apparatus}

The stimuli were created and presented with E-Prime 2.0 Professional (Psychology Software Tools) on an IBMcompatible PC with a 17-in. flat-screen monitor $(1,280 \times 1,024$ pixels). Placed immediately below the monitor was a built-in Tobii T120 eyetracker that measured eye movements of both eyes (measurement error about $0.5^{\circ}$; sampling frequency, $120 \mathrm{~Hz}$ ). A chin-and-forehead rest immobilized the head and controlled the $57-\mathrm{cm}$ viewing distance. The ambient illumination was dim.

\section{Subjects}

Thirty-two naïve students of the University of Padua (16 males, 16 females; average age: 23.8 years) with normal or corrected-to-normal vision participated in return for a small payment that depended on performance. The earnings were increased by an average of 0.11 Euros with each correct response and reduced by 0.10 Euros with each anticipation or missed response deadline; the total pay could reach a maximum of 11.88 Euros. Eye movements during the trials were discouraged, and trials affected by them were discarded, but this had no monetary consequences. For reasons that will be discussed in a dedicated nonmethodological article, the exact payment scheme was varied and counterbalanced between subjects. Here, it suffices to note that the present objective measurement of MIB, unlike previous subjective ones, allows performance manipulation via trial-by-trial monetary rewards.

\section{Stimulus}

Figure 1 and the Supplementary Materials illustrate the stimulus. On each trial, one white and one black disk (the salient and nonsalient targets, respectively; radius, $0.5^{\circ}$; luminances, 120.9 and $11.6 \mathrm{~cd} / \mathrm{m}^{2}$ ) were presented on opposite sides of a red fixation spot (radius, $0.4^{\circ}$; luminance, $120.9 \mathrm{~cd} / \mathrm{m}^{2}$; CIE: $\left.0.6456,0.3342\right)$. The white disk was presented randomly, but equally often, at 1 of 12 evenly distributed locations $4.4^{\circ}$ away from fixation. The gray background $\left(30.4 \mathrm{~cd} / \mathrm{m}^{2}\right)$ contained a $7 \times 7$ pattern $\left(18.2^{\circ} \times 17.3^{\circ}\right)$ of blurred dark-gray crosses (inducers; each $1.6^{\circ} \times 1.2^{\circ}$ and $9.7 \mathrm{~cd} / \mathrm{m}^{2}$ ). This pattern rotated randomly either clockwise or counterclockwise at a speed of 3.48 rotations per second.

After a variable duration (lead time), randomly, one of the targets was physically removed. There were 18 possible lead times that were each presented, at random, an equal number of times during each block of trials. Within each block and lead time condition, black and white targets were removed an equal number of times. The lead times ranged from 1,000 to $7,800 \mathrm{~ms}$, with $400-\mathrm{ms}$ increments. With the present method, guessing is of no concern. Accuracy thus ranges from $0 \%$ to $100 \%$ (as in a yes/no task) and not from a particular guessing rate to $100 \%$ (like the $50 \%-100 \%$ range typical of the two-alternative forced choice task). Instead of presenting 18 different lead times, one could employ a staircase procedure. This should increase data collection efficiency but requires careful consideration of potential guessing effects.

\section{Design}

The experiment consisted of 108 trials, divided into a practice block of 12 trials and three experimental blocks of 36 trials each. Target color (black vs. white) and lead time (18 different durations) were both within-subjects variables.

\section{Procedure}

Each subject underwent a standard 9-point eyetracker calibration procedure, after which the experiment started. During each trial, subjects were to maintain fixation and refrain from blinking (our eyetracker tended to respond to blinks as if they were eye movement errors). During the 2,000-ms intertrial interval, subjects were free to blink and move their eyes, but after this interval, the fixation spot flickered until it was fixated, and a new trial did not start until proper fixation was achieved. The limits on eye movements, although strict, did not seem to burden the subjects much; they enjoyed the experiment as if it were a video game.

The subjects' task was to press the space bar immediately after one of the two targets had been removed from the screen and before an 832-ms response deadline. Correct responses were followed by a short pleasant high-pitched ringing of a bell, anticipations and missed deadlines by a short unpleasant low-pitched alarm, and eye movement errors by a series of short low-pitched beeps. Each trial ended immediately after either a correct response or an error. 
After each experimental block, subjects received feedback on the amount of money won so far, took a brief self-paced break, and then pressed the space bar to continue. At the end of the experiment, the total amount of money won was shown, and the subject was either paid or thanked without pay if the amount was zero or below zero.

\section{Results and discussion}

Figure 2 shows the main result: As was predicted, accuracy in detecting the physical offset of the target decreased dramatically with the time leading up to this event (except for one single missed deadline, all errors that were not due to eye movements were anticipations). With increasing lead time, accuracy first improved slightly and then steadily declined. Future research is needed to determine whether suboptimal preparedness of the subject at trial onset could explain the odd initial improvement or whether it is a genuine characteristic of MIB. The general pattern, however, is clear: Target offset detection accuracy declines with lead time, indicating that it is a valid objective measure of MIB.

With the least-squares method, we fitted both a linear and a logistic curve to the data. The linear one was best, and the proportion of the variance that it explained was large, $R^{2}=.85, p<.001, N=18$. For two reasons, the logistic fit was poor in particular at the two tails of the accuracy distribution: (1) At the shortest lead times, accuracy first improved and only then declined, and (2) at the longest lead times, accuracy did not reach an asymptote. Increasing the subjects' preparedness at trial onset might eliminate the initial improvement, and addition of longer lead times is likely to cause accuracy to reach an asymptote. Both should improve

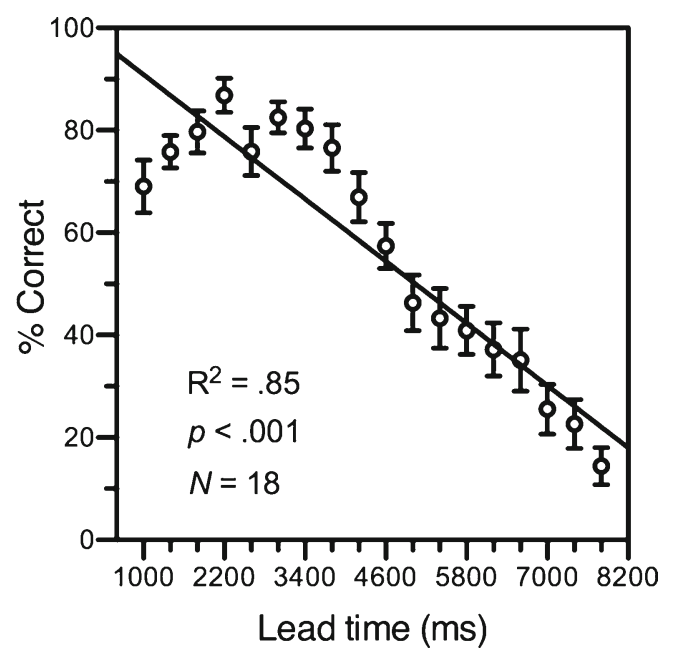

Fig. 2 Target offset detection accuracy as a function of lead time. The diagonal line represents the linear fit to the data. Error bars depict \pm 1 standard error the fits to the data of the logistic function and of S-shaped curves in general.

To obtain reliable fits to the individual data too, more observations per subject are desirable. Nevertheless, consistent with our hypothesis, all the 32 correlations between lead time and accuracy, calculated separately for each of the subjects, were negative, and 29 of them significantly so.

There was an effect of experimental block on accuracy, $F$ $(62)=18.72, p<.0001$; means were $51 \%, 60 \%$, and $63 \%$, respectively. The last two blocks were significantly different from the first block, both $t \mathrm{~s}(31)>4.86, p<.0001$, but not from one another, $t(31)=1.13, p=.27$, suggesting that performance had reached an asymptote.

Figure 3 shows that, as was predicted, accuracy did not increase, but decreased, with target salience. That is, there was more MIB (a lower accuracy) for the salient white targets than for the nonsalient black ones. The counterintuitive effect was small (3.1\%) but significant, one-sample $t$ $(16)=3.29, p=.005$ (excluding one outlying data point that was more than 2.5 standard deviations away from the mean; with the outlier included, the result was marginal: $2.3 \%$, one-sample $t(17)=1.87, p=.08$ ). The greater saliency of the white targets, relative to the black ones, can be seen as the result of the former grouping less well than the latter with the dark-gray inducers (grouping by luminance polarity; Masin, 2003). More radical manipulations of grouping between the two should produce bigger effects (Hsu et al., 2004). In any case, it is overwhelmingly clear that our objective measure, like previous subjective ones, indicate that even quite salient targets are subject to MIB.

\section{Conclusion}

We have presented a method that, for the first time, permits motion-induced blindness to be measured objectively and

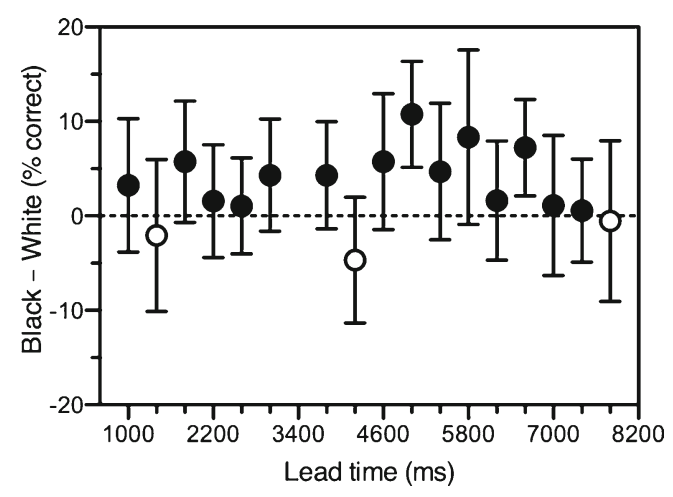

Fig. 3 Difference in accuracy between black and white targets for 17 different lead times (one outlying data point was excluded). Filled symbols represent a greater accuracy for black than for white targets. Open symbols represent the converse. Error bars depict \pm 1 standard error 
with feedback - a method that produces observations that are verifiably either correct or incorrect. This method allows straightforward fitting of psychometric functions, with accuracy data that range from $0 \%$ to $100 \%$ (as in a yes/no task) and not from a guessing rate to $100 \%$ (as in a twoalternative forced choice task). In principle, a staircase extension of the method, for more efficient data collection, is also feasible. It should be possible to apply the method presented here not only in MIB studies, such as the present one, but also in investigations of similar types of temporary blindness that are induced without motion (New \& Scholl, 2008), or even without dynamic inducers of any kind (Gorea \& Caetta, 2009; Hsu, Kramer, \& Yeh, 2010). In sum, the method we propose is objective, very simple, measures what it intends to measure, and produces data that are easy to collect and straightforward to analyze.

Acknowledgments This work was supported by a grant from the University of Padua (Progetto di Ricerca di Ateneo CPDA084849) to Paola Bressan. We are grateful to Marco Zorzi for the use of his eyetracker and laboratory.

\section{References}

Bonneh, Y. S., Cooperman, A., \& Sagi, D. (2001). Motion-induced blindness in normal observers. Nature, 411, 798-801.

Bonneh, Y. S., Donner, T. H., Sagi, D., Fried, M., Cooperman, A., Heeger, D. J., \& Arieli, A. (2010). Motion-induced blindness and microsaccades: Cause and effect. Journal of Vision, 10(14):22, 115.

Bressan, P. \& Kramer, P. (2008). Gating of remote effects on lightness. Journal of Vision, 8(2):16, 1-8.

Donner, T. H., Sagi, D., Bonneh, Y. S., \& Heeger, D. J. (2008). Opposite neural signatures of motion-induced blindness in human dorsal and ventral visual cortex. Journal of Neuroscience, 28, 10298-10310.
Gorea, A. \& Caetta, F. (2009). Adaptation and prolonged inhibition as a main cause of motion-induced blindness. Journal of Vision, 9 (6): 16, 1-17.

Hsu, L.-H., Yeh, S.-L., \& Kramer, P. (2004). Linking motion-induced blindness to perceptual filling-in. Vision Research, 44, 28572866.

Hsu, L.-H., Yeh, S.-L., \& Kramer, P. (2006). A common mechanism for perceptual filling-in and motion-induced blindness. Vision Research, 46, 1973-1981.

Hsu, L.-H., Kramer, P., \& Yeh, S.-L. (2010). Monocular depth effects on perceptual fading. Vision Research, 50, 1649-1655.

Kawabe, T., Yamada, Y., \& Miura, K. (2007). How an abrupt onset cue can disrupt motion-induced blindness. Consciousness \& Cognition, 16, 374-380.

Klotz, W., \& Ansorge, U. (2007). Preceding stimulus awareness augments offset-evoked potentials: Evidence from motion-induced blindness. Psychological Research, 71, 694-702.

Libedinsky, C., Savage, T., \& Livingston, M. (2009). Perceptual and physiological evidence for a role for early visual areas in motioninduced blindness. Journal of Vision, 9(1):14, 1-10.

Masin, S. C. (2003). Effects of algebraic and absolute luminance differences on achromatic surface grouping. Perception, 32, 615-620.

Mitroff, S. R., \& Scholl, B. J. (2004). Seeing the disappearance of unseen objects. Perception, 33, 1267-1273.

Mitroff, S. R., \& Scholl, B. J. (2005). Forming and updating object representations without awareness: Evidence from motioninduced blindness. Vision Research, 45, 961-967.

Montaser-Kouhsari, L., Moradi, F., Zandvakili, A., \& Esteky, H. (2004). Orientation-selective adaptation during motion-induced blindness. Perception, 33, 249-254.

New, J. J., \& Scholl, B. J. (2008). "Perceptual scotomas": A functional account of motion-induced blindness. Psychological Science, 19, $253-259$.

Schölvinck, M. L., \& Rees, G. (2009). Neural correlates of motioninduced blindness in the human brain. Journal of Cognitive Neuroscience, 22, 1235-1243.

Tschacher, W., Schuler, D., \& Junghan, U. (2006). Reduced perception of the motion-induced blindness illusion in schizophrenia. Schizophrenia Research, 81, 261-267.

Wilke, M., Mueller, K.-M., \& Leopold, D. A. (2009). Neural activity in the visual thalamus, reflects perceptual suppression. Proceedings of the National Academy of Science, 106, 9465-9470. 